\title{
Very-High-Energy Gamma-ray observations of X-ray binaries
}

\section{Marc Ribó*}

Universitat de Barcelona, Spain

E-mail: mribo@am.ub.es

\begin{abstract}
The new generation of imaging atmospheric Cherenkov telescopes (HESS, MAGIC, VERITAS) has allowed to conduct sensitive observations in the $\mathrm{TeV}$ regime. Several X-ray binaries have been detected, among them PSR B1259-63, LS 5039, LS I +61 303 and very recently Cygnus X-1. All of them contain high-mass donors. While in the case of PSR B1259-63 the compact object is a confirmed young non-accreting pulsar, and in the case of Cygnus X-1 is a dynamically confirmed stellar-mass black hole and microquasar jet source, the situation is not yet clear in the cases of LS 5039 and LS I +61 303. I summarize here the current status of Very-High-Energy observations of X-ray binaries and discuss possible scenarios that have been put forward to explain the $\mathrm{TeV}$ emission of these systems.
\end{abstract}

Supernovae: lights in the darkness

October 3-5, 2007

Maó (Menorca)

* Speaker. 


\section{Introduction}

There are different astrophysical scenarios in which particles are accelerated up to $\mathrm{TeV}$ energies. Interestingly, these particles can produce gamma-ray photons of Very High Energies (VHE, $E>100 \mathrm{GeV}$ ) that can travel in straight lines from the original sources to the observer, contrary to charged cosmic rays of these energies that are deflected by galactic magnetic fields. Therefore, observational $\mathrm{TeV}$ astronomy can provide very useful information to constrain astrophysical scenarios for particle accelerators. In particular, detailed spectra can shed light on the leptonic or hadronic nature of the accelerated particles that produce the $\mathrm{GeV}-\mathrm{TeV}$ emission, either by inverse Compton scattering of low-energy photons or by neutral pion decay after inelastic proton-proton collisions, respectively.

Although the idea to detect gamma-rays of $\mathrm{TeV}$ energies using the Cherenkov light that they produce when entering the atmosphere was introduced about 50 years ago, it took a few decades of effort to clearly detect the first source, the Crab Nebula in 1989. After a few years of observations with the third generation of imaging atmospheric Cherenkov telescopes, several tens of sources have been detected. Gamma-ray observations in the $\mathrm{TeV}$ regime can certainly be considered nowadays as a new branch of observational astronomy.

An X-ray binary is a binary system containing a compact object, either a neutron star or a stellar-mass black hole accreting, or interacting, with the matter from the companion star in such a way that produces X-ray emission. The most recent catalogs contain a total of 300 galactic X-ray binaries ([26], [27]). In 114 of such systems the companion star has a spectral type $\mathrm{O}$ or B, and they are classified as High Mass X-ray Binaries (HMXBs), where mass transfer takes place via a decretion disk (in Be stars) or via a strong stellar wind or Roche-lobe overflow (in OB supergiants). In $186 \mathrm{X}$-ray binaries the optical companion has a spectral type later than $\mathrm{B}$, and they are called Low Mass X-ray Binaries (LMXBs), where mass transfer occurs via Roche-lobe overflow. More than $50 \mathrm{X}$-ray binaries have displayed synchrotron radio emission produced by accelerated particles with energies following a non-thermal distribution. About 15 of them have shown extended radio emission in the form of jets. The systems in which large proper motions, indicative of relativistic speeds, have been measured within the jets, have been called microquasars (see [31] and [17] for reviews on the topic). Interestingly, four radio-emitting X-ray binary systems have been detected in VHE gamma rays.

Here I shortly review the current $\mathrm{TeV}$ instrumentation (Sect. 2), the observations of $\mathrm{TeV}$ emitting X-ray binaries (Sect. 3) and the searches for new such systems (Sect. 4). I finish with some comments on future instrumentation (Sect. 5) and with the conclusions (Sect. 6).

\section{Current TeV instrumentation}

VHE gamma-ray astronomy in the $\mathrm{TeV}$ range is conducted using ground-based imaging atmospheric Cherenkov telescopes (IACTs). A detailed description of the early history of the atmospheric Cherenkov technique can be found in [46]. The first TeV source to be discovered was the Crab Nebula, which was detected at $9.0 \sigma$ with the Whipple $10 \mathrm{~m}$ telescope equipped with a 37-pixel camera on the focal plane ([47]; after 20 years of searches!). About 75 sources have been 


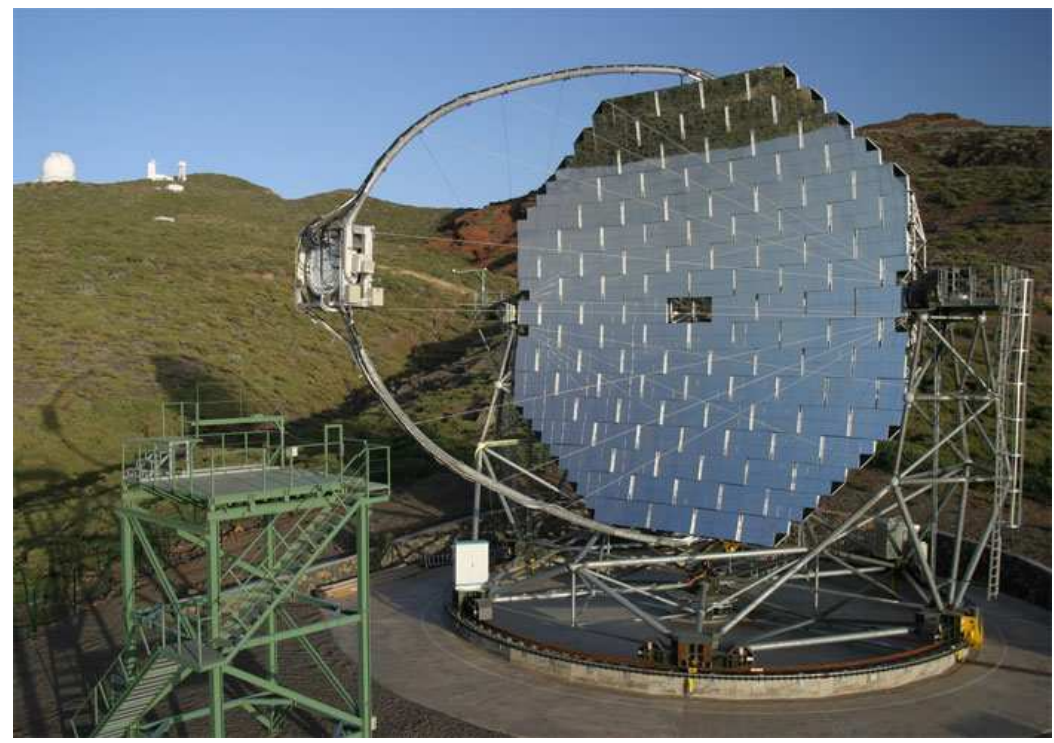

Figure 1: The MAGIC telescope at the Observatorio del Roque de los Muchachos, in the Canary Island of La Palma. With its $17 \mathrm{~m}$ diamater aperture MAGIC is currently the largest single-dish Cherenkov telescope in the world.

discovered since then thanks to the development of instrumentation and the consequent gain in sensitivity (see Robert Wagners' http: / /www . mppmu.mpg. de/ rwagner/sources/).

There are currently four IACTs of third generation:

- CANGAROO-III (Collaboration of Australia and Nippon for a GAmma Ray Observatory in the Outback $)^{1}$

- HESS (High Energy Stereoscopic System) ${ }^{2}$

- MAGIC (Major Atmospheric Gamma Imaging Cherenkov) ${ }^{3}$ (see Fig. 1)

- VERITAS (Very Energetic Radiation Imaging Telescope Array System) ${ }^{4}$

Their main properties are listed in Table 1. As it can be seen, CANGAROO-III and HESS are located in the Southern Hemisphere, while MAGIC and VERITAS are placed in the Northern Hemisphere. This allows for a double check of some of the most southern or northern sources, always welcome in $\mathrm{TeV}$ astrophysics. CANGAROO-III, due to its low elevation, has a high threshold of about $500 \mathrm{GeV}$. The other instruments can reach the $100-200 \mathrm{GeV}$ regime and even lower in the case of MAGIC, thanks to its huge dish and the high elevation of the site. All these IACTs have to be pointed to the specific regions of the sky to be studied.

HESS started stereoscopic observations in 2003, MAGIC normal operations in 2004 and CANGAROO-III in 2005, while VERITAS started stereoscopic observations at the end of 2006

\footnotetext{
${ }^{1}$ http://icrhp9.icrr.u-tokyo.ac.jp/index.html

${ }^{2}$ http: //www.mpi-hd.mpg.de/hfm/HESS/

${ }^{3}$ http: //wwwmagic.mppmu.mpg.de/

${ }^{4}$ http: //veritas.sao.arizona.edu/
} 


\begin{tabular}{lllllll}
\hline IACT & Location & $\begin{array}{l}\text { Elevation } \\
{[\mathrm{km}]}\end{array}$ & Telescopes & $\begin{array}{l}\text { Aperture } \\
{[\mathrm{m}]}\end{array}$ & $\begin{array}{l}\text { FOV } \\
{\left[{ }^{\circ}\right]}\end{array}$ & $\begin{array}{l}\text { Energy } \\
{[\mathrm{GeV}]}\end{array}$ \\
\hline CANGAROO-III & Woomera, Australia & 0.2 & 4 & 10 & 4.0 & $>500$ \\
HESS & Gamsberg, Namibia & 1.8 & $4(+1)$ & $12(28)$ & 5.0 & $>100$ \\
MAGIC & La Palma, Spain & 2.2 & $1(+1)$ & $17(17)$ & 3.5 & $>60$ \\
VERITAS & Arizona, USA & 1.3 & 4 & 12 & 3.5 & $>100$ \\
\hline
\end{tabular}

Table 1: Third Generation of Imaging Atmospheric Cherenkov Telescopes (IACTs).

and has just completed the array in 2007. The most interesting results published up to now in the field of X-ray binaries have been provided by HESS and MAGIC, and I will concentrate on them. As a reference, the flux sensitivity at the $5 \sigma$ level in $50 \mathrm{~h}$ of observations is $\simeq 2 \%$ of the Crab Nebula at $250 \mathrm{GeV}$ with MAGIC and $\simeq 1 \%$ with HESS.

\section{TeV emitting X-ray binaries}

Four X-ray binaries have been detected up to now at TeV energies: PSR B1259-63 [1], LS 5039 [2], LS I +61 303 [5] and recently Cygnus X-1 [6]. All of them are high mass X-ray binaries (HMXBs), where a huge photon field is available for inverse Compton (IC) scattering. On the other hand, a decretion disk might exist, thus providing targets for $p p$ collisions and $\pi^{0}$ decay. It is worth to mention that low mass X-ray binaries (LMXBs) tend to be transient sources and, provided they were able to produce $\mathrm{TeV}$ photons, one should observe at the right time to detect them.

\subsection{PSR B1259-63}

Located at a distance of $1.5 \mathrm{kpc}$, PSR B 1259-63 is composed of a $48 \mathrm{~ms}$ radio pulsar orbiting a B2 Ve star every $1237 \mathrm{~d}$ in a highly eccentric orbit with $e=0.87$ [22]. A sketch of this binary system can be seen in Fig. 2. The pulsed radio emission is not detected when the neutron star is behind the decretion disk of the companion [21], and two outbursts of optically thin synchrotron radio emission are seen before and after periastron passage, in coincidence with the decretion disk crossing [23]. This binary system was discovered at TeV energies by the HESS Collaboration, who reported variable fluxes during the 2004 periastron passage [1]. The observed spectrum can be fit with a simple power law at all epochs, with photon index $\Gamma=2.7$ above $380 \mathrm{GeV}$ (up to $\sim 3 \mathrm{TeV}$ ). However, the lightcurve is puzzling when compared to previously available models, as can be seen in Fig. 3 [1].

Although it is clear that the interaction between the relativistic wind of the young non-accreting pulsar and the polar wind and/or the equatorial (but inclined) decretion disk of the donor star plays a major role, the exact mechanism producing the $\mathrm{TeV}$ emission is not known. Pure leptonic scenarios invoking IC scattering of UV photons from the star by relativistic electrons from the pulsar wind have been put forward, although key parameters remain unconstrained due to systematic and statistical uncertainties in the TeV lightcurve [24]. On the other hand, hadronic scenarios where the $\mathrm{TeV}$ emission is produced by $\pi^{0}$ decay, after inelastic $p p$ collisions, and radio/X-ray emission by 


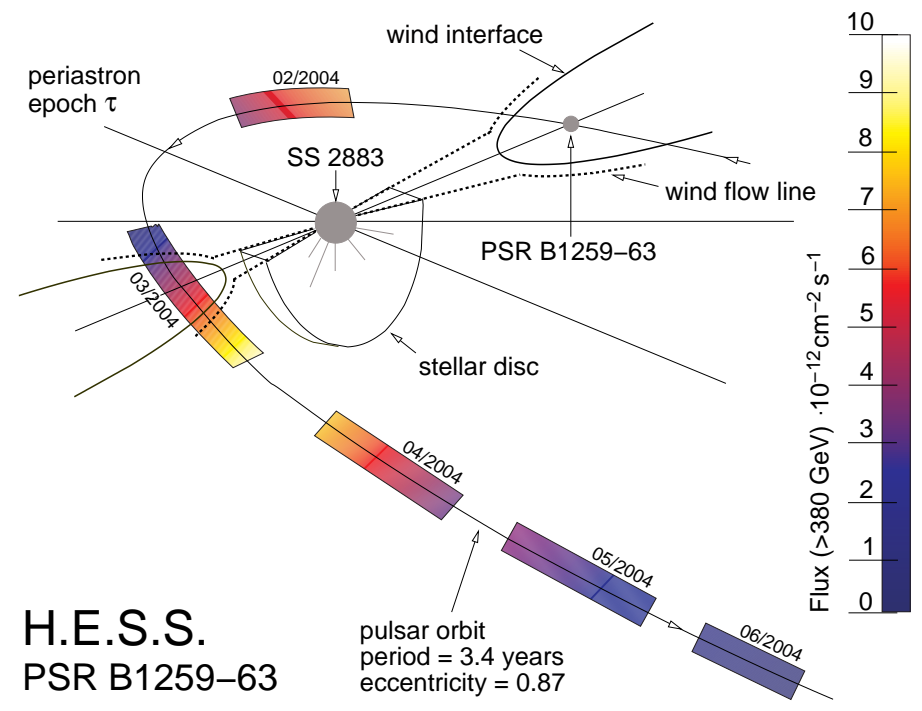

Figure 2: Sketch of the orbit of PSR B1259-63 with respect to the line of sight. The orientation of the disk with respect to the orbital plane is not precisely known. The colour gradient bars along the orbit indicate the periods of HESS observations and show the integral TeV flux using a smoothed light curve. From [1].

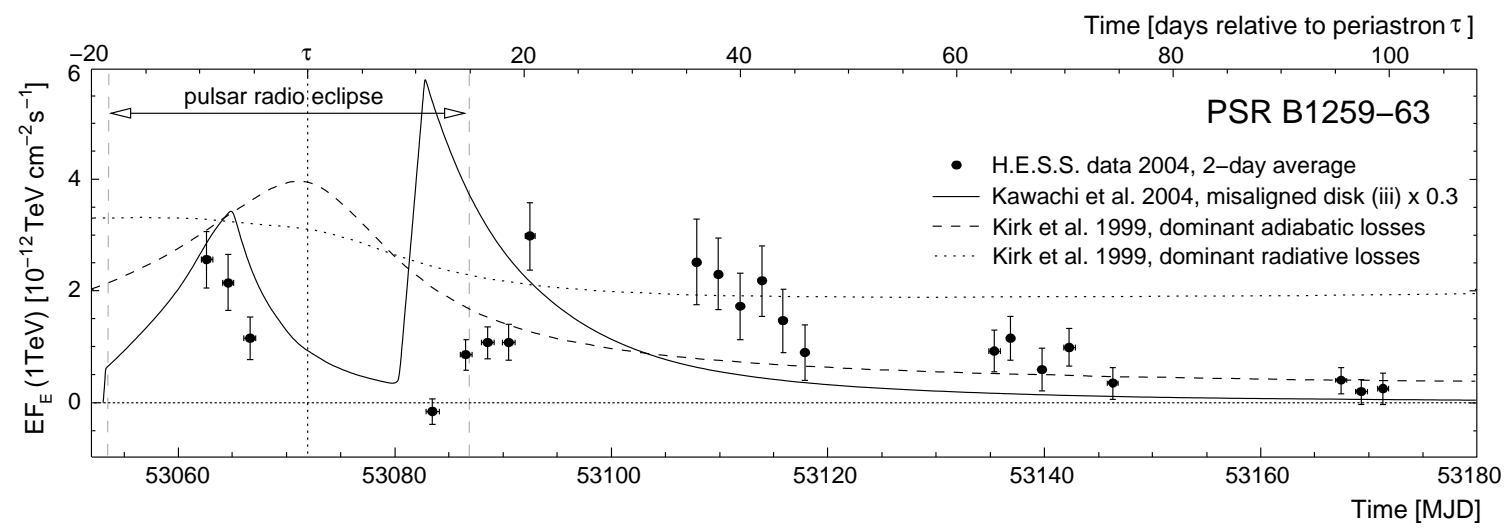

Figure 3: Comparison of the light curves predicted by different models of VHE $\gamma$-ray emission with the corresponding light curve obtained from the HESS data using a bin width of 2 days. From [1].

$\pi^{ \pm}$decay and IC scattering have also been proposed [32]. Observations in the GeV range by the GLAST satellite, to be launched around May 2008, should provide the answer to this open question.

\subsection{LS I +61 303}

Located at $2.0 \mathrm{kpc}$ from us, LS I +61303 is composed of a rapidly rotating early type B0 Ve star with a stable equatorial decretion disk and mass loss, and a compact object with a mass between 1 and $4 \mathrm{M}_{\odot}$ orbiting it every $\sim 26.5 \mathrm{~d}$ (see [10] and references therein). The TeV emission detected by MAGIC is highly variable: upper limits have been found during periastron passage and a peak occurs near apastron, suggesting periodic emission ([5]; see Fig. 4). The spectrum can always be fitted with a simple power law with photon index $\Gamma=2.6$ from $200 \mathrm{GeV}$ to $5 \mathrm{TeV}$ [42]. Recent observations by the MAGIC and VERITAS collaborations clearly indicate the existence of 


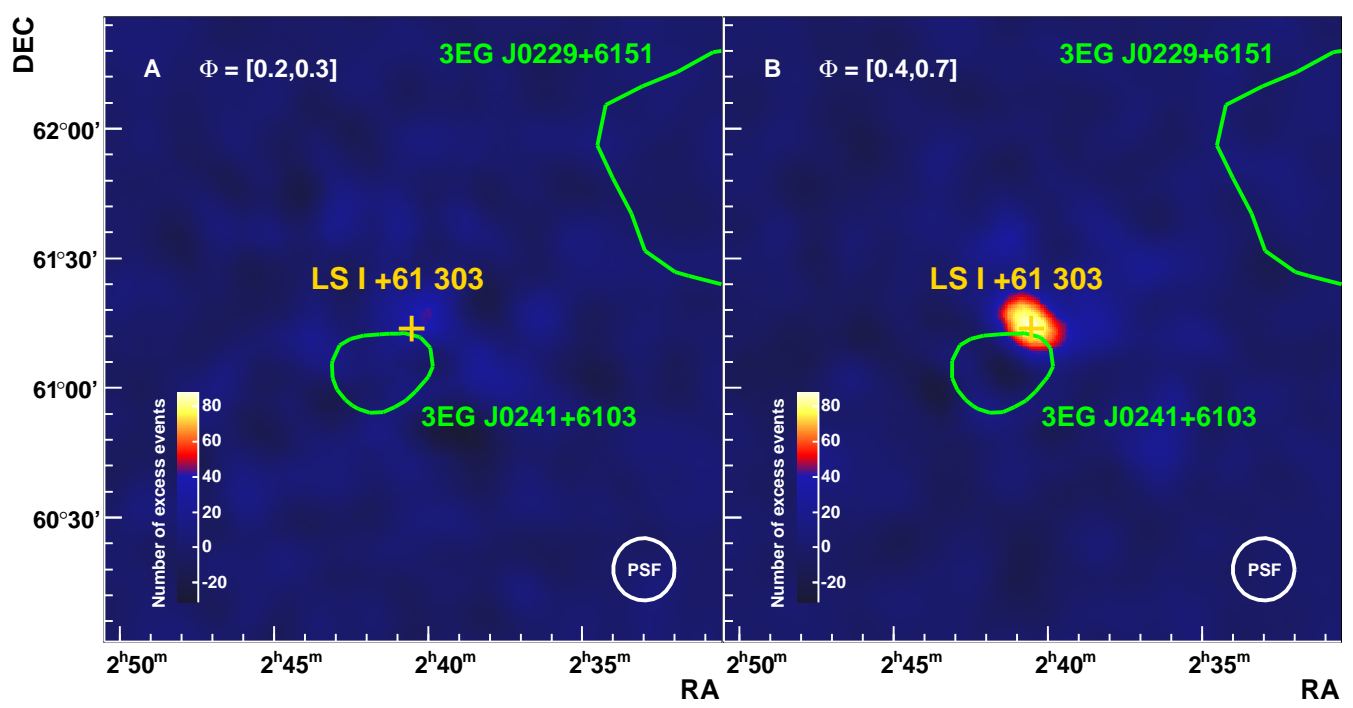

Figure 4: Smoothed VHE sky maps of LS I +61 303 in the orbital phase range $0.2-0.3$ close to periastron passage (left) and in phase range $0.4-0.7$ near apastron (right). The variability is apparent. From [5].

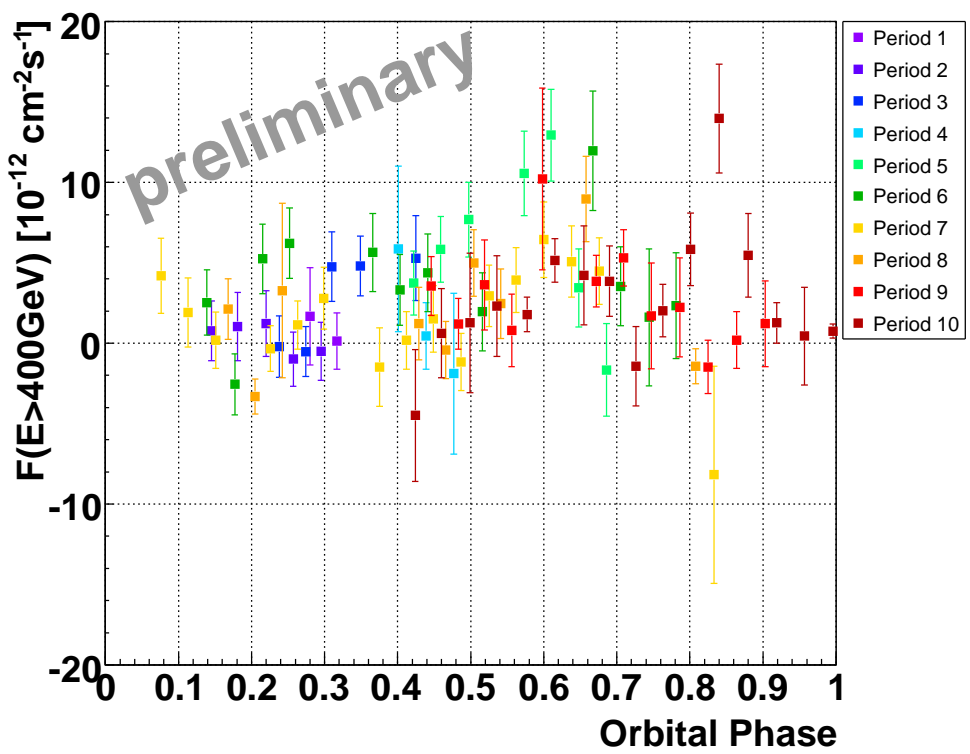

Figure 5: Lightcurve of the integral gamma-ray flux of LS I +61 303 for energies above $400 \mathrm{GeV}$ folded using the orbital period of $26.4960 \mathrm{~d}$. Each color represents a different orbital cycle observed. From [42].

an orbital TeV variability in LS I +61 303 ([42], see Fig. 5, and [28], respectively). The detection at TeV energies confirms the association of LS I +61 303 with the GeV COS-B (EGRET) source 2CG 135+01 (3EG 0241+6103) [20, 19].

Massi and collaborators reported the discovery of an extended jet-like and apparently precessing radio emitting structure at angular extensions of 10-50 milliarcseconds [30]. Due to the presence of (apparently relativistic) radio emitting jets, LS I +61 303 was proposed to be a microquasar. However, recent VLBA images obtained during a full orbital cycle show a rotating elongated morphology that Dhawan and collaborators interpreted in the context of the interaction 

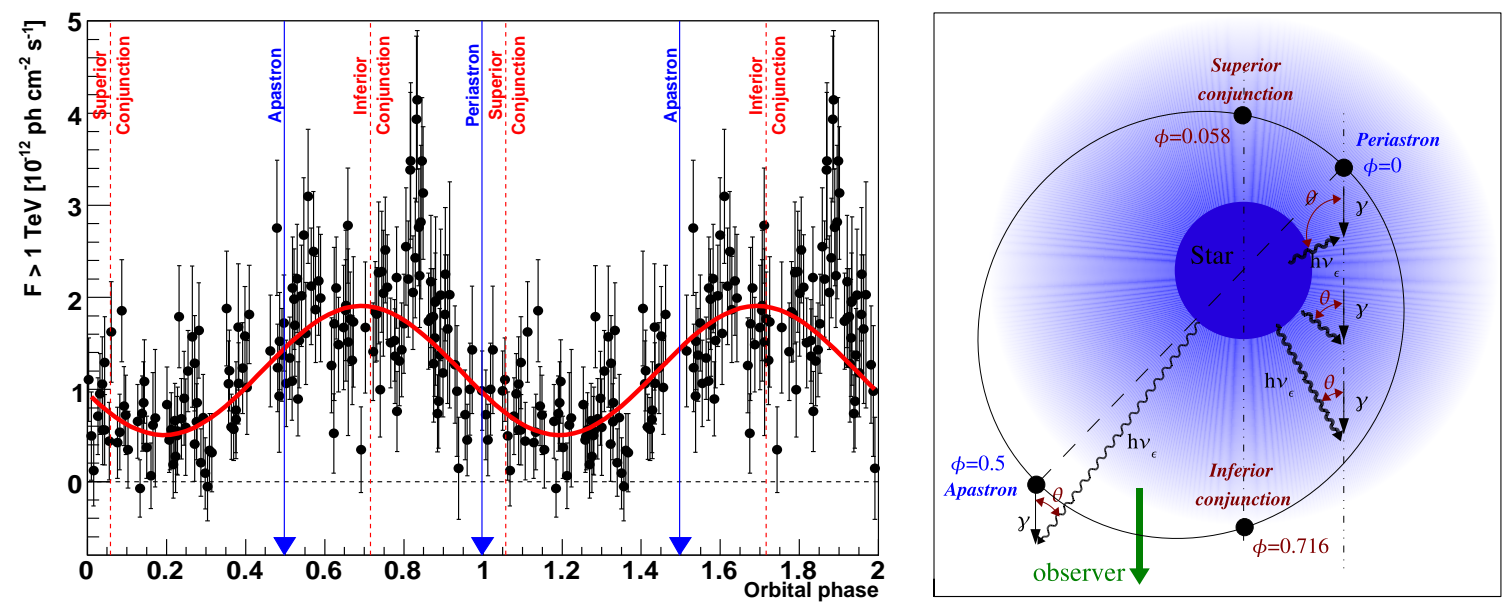

Figure 6: Left: VHE gamma-ray emission of LS 5039 detected by HESS folded with its $3.9 \mathrm{~d}$ orbital period. A clear quasi-sinusoidal variability is seen. Right: geometry of the binary system orbit as seen from above. VHE $\gamma$-rays (straight black lines with arrows) can be absorbed by optical photons of energy $h v_{\varepsilon}$, when their scattering angle $\theta$ exceeds zero. From [3].

between the wind of the companion and the relativistic wind of a young non-accreting ms pulsar, similarly as in PSR B1259-63 ([12]; see [15] for details about the model). The pulsed radio emission would not be detected because of free-free absorption. However, recent detailed simulations of this pulsar-wind interaction reveal a problem in this scenario: to explain the observed GeV luminosity the spin-down power of the putative pulsar should be so high that the wind of the companion could not collimate the radio emitting particles [40]. The nature of the source is thus still a matter of debate.

\subsection{LS 5039}

Located at a distance of $2.5 \mathrm{kpc}$, LS 5039 contains a compact object of unknown nature, with mass between 1.4 and $5 \mathrm{M}_{\odot}$, orbiting every 3.9 days an $\mathrm{ON} 6.5 \mathrm{~V}((\mathrm{f}))$ donor in a slightly eccentric orbit [11]. This binary system is escaping from the Galactic plane with a runaway velocity of more than $100 \mathrm{~km} \mathrm{~s}^{-1}[38,11]$. The TeV flux of this binary system as seen by HESS is clearly periodic, with enhanced emission at inferior conjunction of the compact object (see Fig. 6) and a clear variability in the $\mathrm{TeV}$ spectrum between inferior and superior conjunction [3]. This suggests that photon-photon absorption (which has an angle dependent cross-section) within the binary system plays a major role. However, the non-zero flux observed at superior conjunction of the compact object and the lack of variability at $\sim 200 \mathrm{GeV}$ argues against this simple interpretation, and suggests that there may be an orbital phase-dependent electron acceleration and/or that the $\mathrm{TeV}$ emission could be produced far from the compact object (see e.g. [25]). The discovery of LS 5039 as a TeV emitter confirmed its association with the GeV EGRET source 3EG 1824-1514 [34].

The detection of elongated asymmetric emission in high-resolution radio images obtained with the VLBA and the EVN was interpreted as evidence of its microquasar nature, and suggested that the source was persistently producing mildly relativistic ejections with a velocity of $\sim 0.15 c$ $[34,35]$. Although the X-ray spectra are compatible with those of accreting black holes while they are in the so-called low/hard state [8], the radio spectra are optically thin with a spectral index 

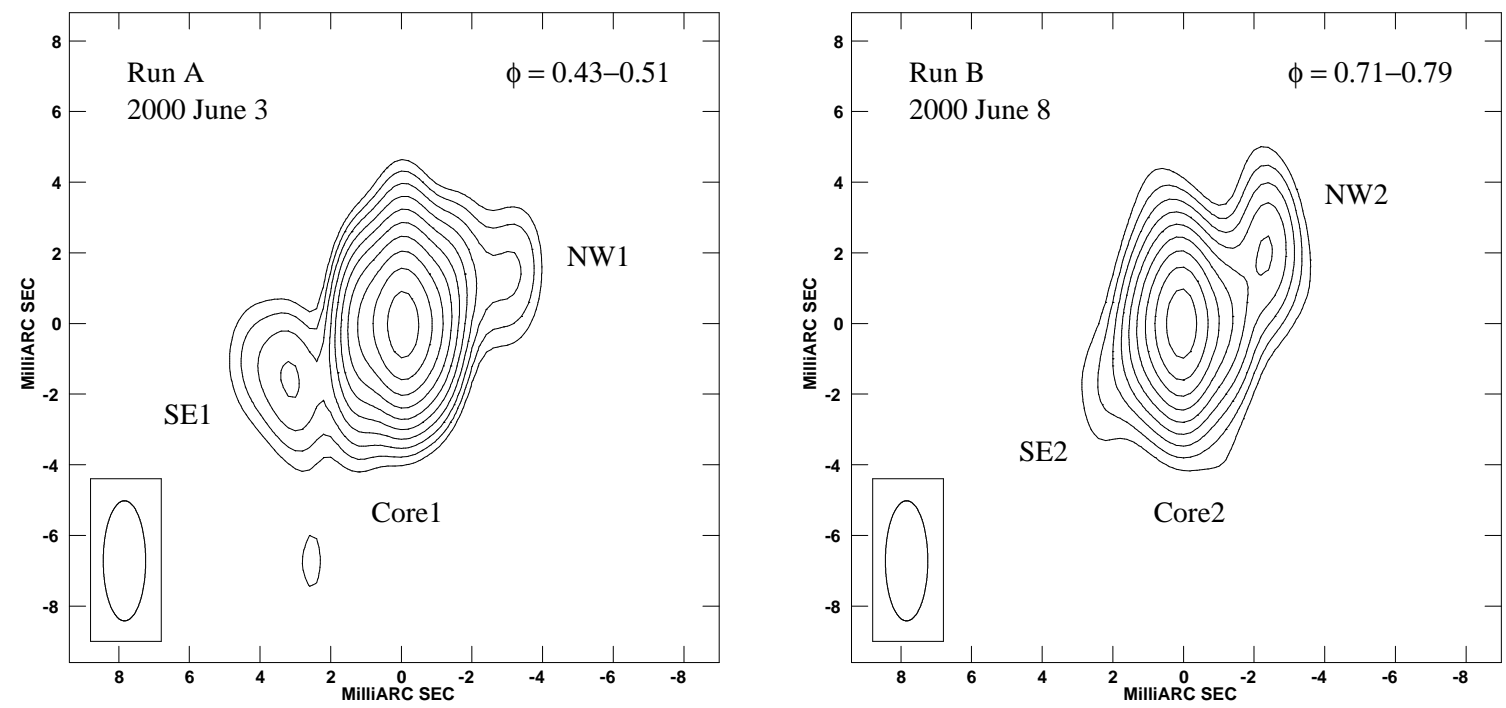

Figure 7: VLBA self-calibrated images of LS 5039 at $5 \mathrm{GHz}$. The dates and orbital phases are quoted in the images. There is extended radio emission that appears nearly symmetric for run A and clearly asymmetric for run B, with a small change of $\sim 10^{\circ}$ in its position angle. From [39].

of -0.5 [29, 37]. Theoretical modeling in the microquasar scenario, where the $\mathrm{TeV}$ emission is produced by IC up-scattering of stellar UV photons by relativistic electrons in the jet [9], has allowed to reproduce the observed SED from radio to VHE gamma-rays [36]. However, the lack of clear accretion signatures and the similarities with the SEDs of PSR 1259-63 and LS I +61 303 has led other authors to model its multi-wavelength emission using the scenario of wind interactions [15]. One of the predictions of this kind of modeling is the periodic change in the direction and shape of the extended radio morphology, as well as in the peak position of the radio core, depending on the orbital phase. Therefore, high resolution VLBI observations can help to unveil the nature of the system. In this context, new VLBA radio images of the source (see Fig. 7) indicate that a simple and shockless microquasar scenario cannot easily explain the observed changes in morphology, because it requires a jet precession that is in contrast with the persistency of position angle of the extended emission [39]. On the other hand, an interpretation within the young non-accreting pulsar scenario requires the inclination of the binary system to be very close to the upper limit imposed by the absence of X-ray eclipses. The nature of the source is still an open issue.

The interested reader can find details about the recent modelling of the $\mathrm{TeV}$ lightcurve and spectra of the source using different scenarios in several papers [43, 25, 16, 33].

\subsection{Cygnus X-1}

The $\mathrm{X}$-ray binary Cygnus $\mathrm{X}-1$ is the most recent addition to the selected group of $\mathrm{TeV}$ emitting $\mathrm{X}$-ray binaries [6]. Located at a distance of $2.2 \mathrm{kpc}$, Cygnus X-1 contains an O9.7 Iab donor and an accreting black hole of at least $10 \mathrm{M}_{\odot}$ orbiting it every 5.6 days in a circular orbit (see [48] and references therein). It shows nearly persistent radio emission, which sometimes has been resolved in jet-like features that reveal its microquasar nature [45]. A ring-like structure at arcminute scales has been detected, showing the strong influence of the jet into its surrounding ISM [18]. Cygnus X1 was detected by MAGIC only in a short $\sim 80$ minute time interval with a soft spectrum $(\Gamma \simeq 3.2)$ 


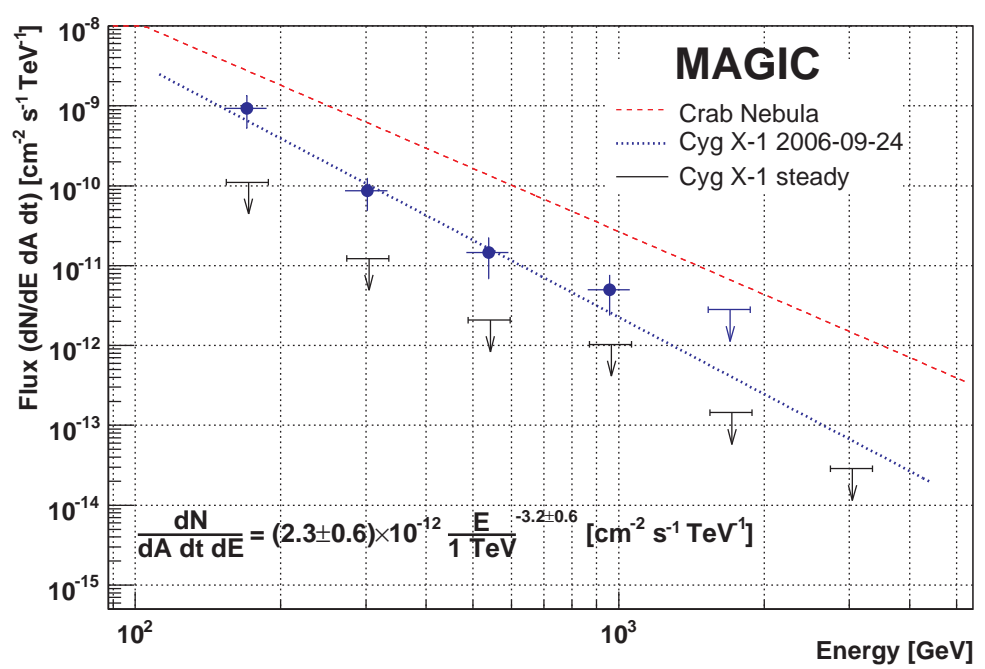

Figure 8: Differential energy spectrum from Cygnus X-1 corresponding to 78.9 minutes of observations between MJD 54002.928 and 54002.987 (2006-09-24). Also shown are the Crab nebula spectrum, the best fit of a power-law to the data, and the $95 \%$ confidence level upper limits to the steady $\gamma$-ray flux. From [6].

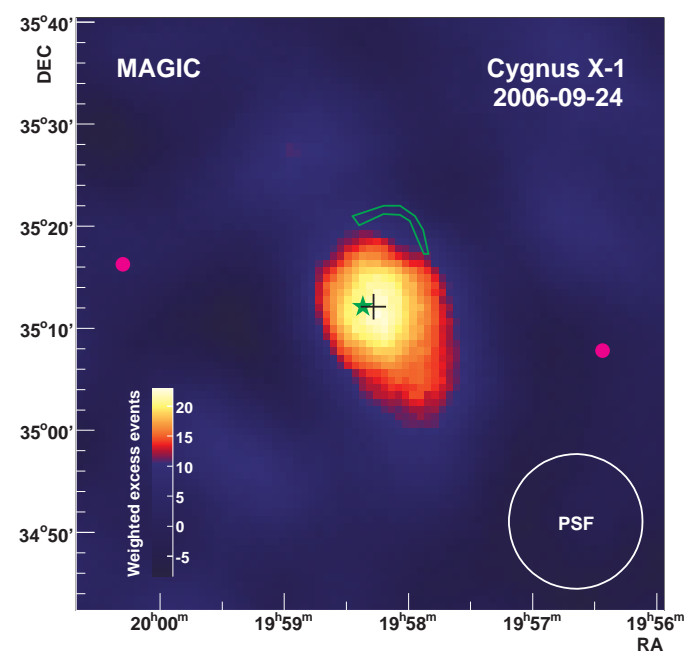

Figure 9: Smoothed VHE sky map above $150 \mathrm{GeV}$ around Cygnus $\mathrm{X}-1$ corresponding to $\sim 80$ minutes of observations on 2006 September 24. The black cross shows the best-fit position of the $\gamma$-ray source. The position of the X-ray source and radio emitting ring-like are marked by the green star and contour, respectively. The purple dots mark the directions tracked during the observations. From [6].

extending only up to $\sim 1 \mathrm{TeV}$, while upper limits are obtained for the rest of the $\sim 40 \mathrm{~h}$ observations (see Fig. 8).

The position of the $\mathrm{TeV}$ emission is compatible with that of Cygnus $\mathrm{X}-1$, excluding the ringlike structure (see Fig. 9). The TeV excess was found at orbital phase 0.91, when the black hole is behind the star and photon-photon absorption should be huge. For instance, [7] computed the opacity to pair production for different injection distances from the center of the massive star and angles of propagation, finding that photons propagating through the intense stellar field towards the observer would find in their way opacities of about 10 at $1 \mathrm{TeV}$. An origin in the jet of this 

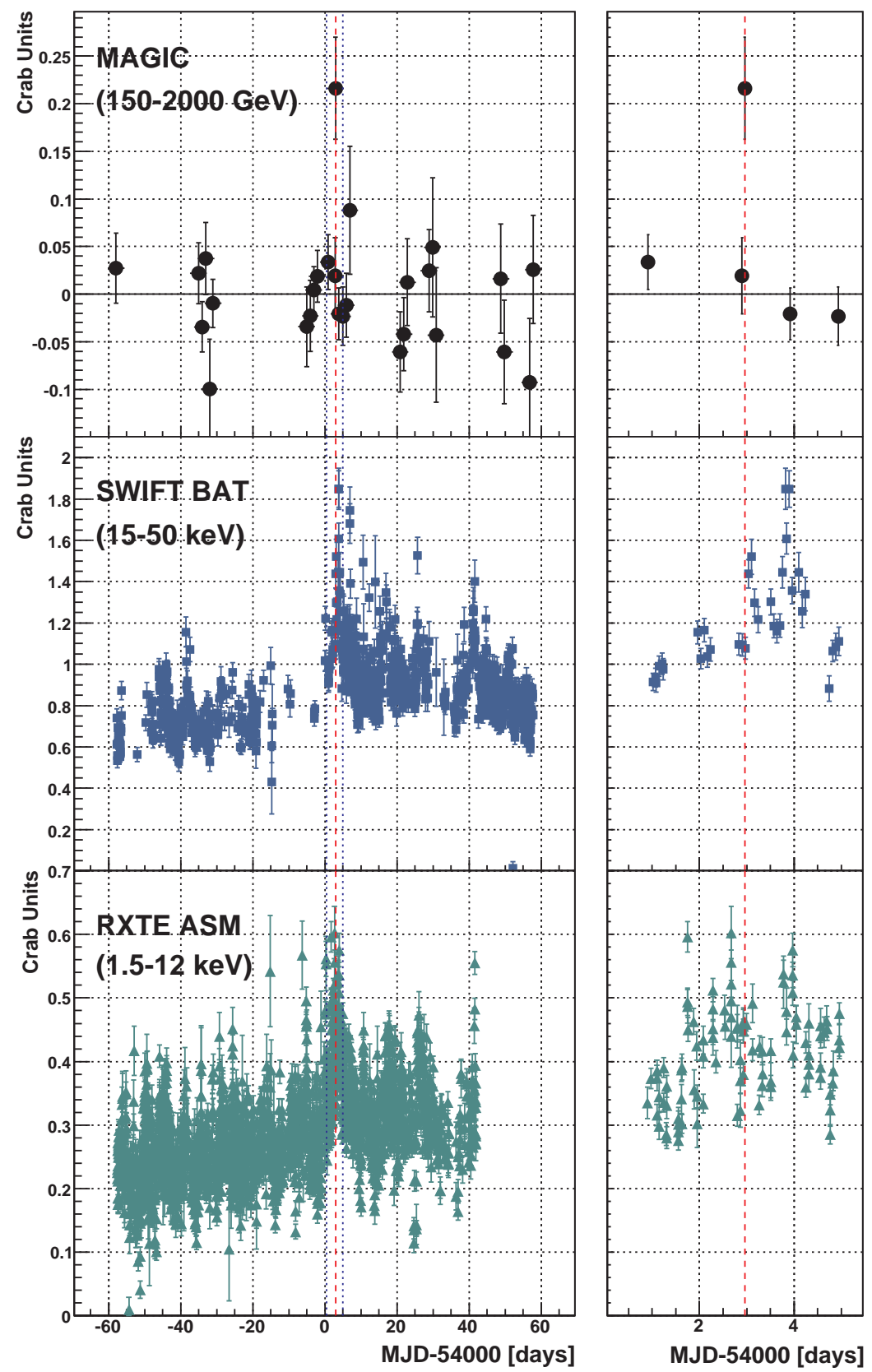

Figure 10: From top to bottom: MAGIC, Swift/BAT and RXTE/ASM measured fluxes from Cygnus X-1 as a function of time. The left panels show the whole time spanned by the MAGIC observations. The vertical, dotted blue lines delimit the range zoomed in the right panels. The vertical red line marks the time of the MAGIC signal. From [6].

microquasar and away from the compact object would relax these values.

The TeV detection took place during a particularly bright hard X-ray state of Cygnus X-1 (see Fig. 10). Simultaneous hard X-ray observations by Swift/BAT in the $15-50 \mathrm{keV}$ energy range reveal that the $\mathrm{TeV}$ excess was found right before the onset of a hard $\mathrm{X}$-ray peak. Observations one day later reveal that no $\mathrm{TeV}$ excess was found during the maximum and decay phase of another 
hard X-ray peak. Although one could speculate with the limited available data, more simultaneous multi-wavelength observations are necessary to build reliable models. In any case, this is the first experimental evidence of VHE emission from a stellar-mass black hole, and therefore from a confirmed accreting X-ray binary.

\subsection{TeV flares in X-ray binaries?}

The TeV lightcurve of LS 5039 shows a pronounced maximum around phase 0.85 (see Fig. 6left). Although it could be interpreted as a TeV flare, it appears to be an orbital feature $[3,33]$. However, it is interesting to note that, apart from the TeV flare of Cygnus X-1, a possible flare has also been observed at phase $\sim 0.85$ in LS I +61 303, as can be seen in Fig. 5 [42]. Therefore, TeV flares could be common phenomena in X-ray binaries. In this context it is worth to note that the low mass X-ray binary and microquasar system GRS $1915+105$ was reported to show a possible flare at a level of $3.1 \sigma$ by the Whipple collaboration [41]. Therefore, new microquasars could be eventually detected while flaring.

\section{The search for new TeV emitting X-ray binaries}

Two X-ray binary systems have been proposed as counterparts to unidentified VHE sources: IGR J16320-4751 for HESS J1632-478 and IGR J16358-4726 for HESS J1634-472 [4]. However, their extended $\mathrm{TeV}$ emission appears to rule out these associations.

The HESS Collaboration has recently reported on two different searches for X-ray binaries. On the one hand, they have searched for $\mathrm{TeV}$ sources at the positions of known X-ray binaries within their Galactic Plane scan, which now amounts to $\sim 230 \mathrm{~h}$. The fields of $29 \mathrm{X}$-ray binaries have been covered, although some of them are affected by nearby TeV sources [13]. Upper limits at around $2 \%$ of the Crab Nebula flux have been obtained for $18 \mathrm{X}$-ray binaries (6 HMXBs, 12 LMXBs). However, I note that 7 out of the 8 observed HMXBs are transient sources, while this is the case for all observed LMXBs. Thus, it is not strange that none of them has been detected if observed at a random time.

On the other hand, HESS has also conducted a systematic search for TeV emission from known X-ray binaries with properties similar to LS 5039 and LS I +61 303 ( $P_{\text {orb }}=3-20$ d, radio jets, etc.; [14]). 11 sources have been observed (6 HMXBs, 5 LMXBs), and upper limits have been found for 10 of them. However, all of the observed LMXBs are transients. In addition, only 2 of the observed HMXBs are closer than $5 \mathrm{kpc}$ : Vela X-1 and $4 \mathrm{U} 1700-37$, where photon-photon absorption by pair creation must be very important and huge, respectively, easily preventing their detection.

\section{The future}

Regarding the near future, it should be noted that the second $17 \mathrm{~m}$ diameter MAGIC telescope will start operations at the end of 2008 (MAGIC-II). In a few years, a huge $28 \mathrm{~m}$ diameter telescope will join the HESS array (HESS-II). At lower energies, the GLAST satellite, to be launched in mid 2008, should provide a daily all-sky survey, that could be used to trigger $\mathrm{TeV}$ observations of flaring X-ray binaries. These facilities will certainly help us to better understand the physics of $\mathrm{TeV}$ emitting X-ray binaries in the near future. 
On the other hand, given the success of instruments like HESS and MAGIC, in the longer term the $\mathrm{TeV}$ community wants to improve the sensitivity by up to a factor of $\sim 10$ and increase the energy range to cover from $\sim 30 \mathrm{GeV}$ to $\sim 100 \mathrm{TeV}$. In this context, a new Cherenkov telescope is being designed: the Cherenkov Telescope Array ${ }^{5}$ (CTA). The CTA will allow to conduct population studies of $\mathrm{TeV}$ emitting $\mathrm{X}$-ray binaries, as well as very sensitive observations to test proposed models. It is important to note here that a guest observer program is planned for CTA.

\section{Conclusions}

The third generation of IACTs has revealed several tens of sources in the TeV sky. Among them, four X-ray binaries have been detected. PSR B1259-63 contains a young non-accreting pulsar, and the interaction between its relativistic wind and the wind or the decretion disk of the companion is behind the $\mathrm{TeV}$ emission. $\mathrm{A} \mathrm{TeV}$ flare has been observed in the microquasar source and dynamically confirmed accreting stellar-mass black hole Cygnus X-1. The situation is not yet clear for the sources LS 5039 and LS I +61 303, although VLBI observations suggest they might contain a young non-accreting pulsar. New instruments like MAGIC-II, HESS-II, and CTA will allow us to conduct population studies of $\mathrm{TeV}$ emitting X-ray binaries in the near (and mid-term) future, while $\mathrm{GeV}$ satellites like GLAST will allow us to disentangle the different proposed models. In conclusion, X-ray binaries are starting to shine in the TeV sky, which is revealing new and very interesting laboratories for astrophysics.

\section{Acknowledgments}

The author acknowledges support by DGI of the Spanish Ministerio de Educación y Ciencia (MEC) under grant AYA2007-68034-C03-01 and FEDER funds. He also acknowledges financial support from MEC through a Ramón y Cajal fellowship. This research has made use of the NASA's Astrophysics Data System Abstract Service, and of the SIMBAD database, operated at CDS, Strasbourg, France.

\section{References}

[1] Aharonian, F., Akhperjanian, A. G., Aye, K.-M., et al. 2005a, A\&A, 442, 1

[2] Aharonian, F., Akhperjanian, A. G., Aye, K.-M., et al. 2005b, Science, 309, 746

[3] Aharonian, F., Akhperjanian, A. G., Bazer-Bachi, A. R., et al. 2006a, A\&A, 460, 743

[4] Aharonian, F., Akhperjanian, A. G., Bazer-Bachi, A. R., et al. 2006b, ApJ, 636, 777

[5] Albert, J., Aliu, E., Anderhub, H., et al. 2006, Science, 312, 1771

[6] Albert, J., Aliu, E., Anderhub, H., et al. 2007, ApJ, 665, L51

[7] Bednarek, W., \& Giovannelli, F. 2007, A\&A, 464, 437

[8] Bosch-Ramon, V., Paredes, J. M., Ribó, M., et al. 2005, ApJ, 628, 388

[9] Bosch-Ramon, V., Romero, G. E., \& Paredes, J. M. 2006, A\&A, 447, 263

\footnotetext{
$5_{\text {http: }} / /$ www.mpi-hd.mpg.de/hfm/CTA/
} 
[10] Casares, J., Ribas, I., Paredes, J. M., Martí, J., \& Allende Prieto, C. 2005a, MNRAS, 360, 1105

[11] Casares, J., Ribó, M., Ribas, I., et al. 2005b, MNRAS, 364, 899

[12] Dhawan, V., Mioduszewski, A., \& Rupen, M. 2006, PoS, Proceedings of the VI Microquasar Workshop: Microquasars and Beyond, ed. T. Belloni, p.52.1

[13] Dickinson, H., Latham, I., Chadwick, P., et al. (for the HESS Collaboration) 2007a, Proc. of the 30th International Cosmic Ray Conference (Mérida), in press, [arXiv: $0710.4057 v 1]$, p. 61

[14] Dickinson, H., Latham, I., Chadwick, P., et al. (for the HESS Collaboration) 2007b, Proc. of the 30th International Cosmic Ray Conference (Mérida), in press, [arXiv: $0710.4057 v 1]$, p. 65

[15] Dubus, G. 2006, A\&A, 456, 801

[16] Dubus, G., Cerutti, B., \& Henri, G. 2008, A\&A, 477, 691

[17] Fender, R. P. 2006, in Compact Stellar X-Ray Sources, ed. W. H. G. Lewin \& M. van der Klis (Cambridge University Press) [arXiv: astro-ph/0303339]

[18] Gallo, E., Fender, R. P., Kaiser, C., et al. 2005, Nature, 436, 819

[19] Hartman, R. C., Bertsch, D. L., Bloom, S. D., et al. 1999, ApJS, 123, 79

[20] Hermsen, W., Swanenburg, B. N., Bignami, G. F., et al. 1977, Nature, 269, 494

[21] Johnston, S., Manchester, R. N., Lyne, A. G., et al. 1992, ApJ, 387, L37

[22] Johnston, S., Manchester, R. N., Lyne, A. G., Nicastro, L., \& Spyromilio, J. 1994, MNRAS, 268, 430

[23] Johnston, S., Ball, L., Wang, N., \& Manchester, R. N. 2005, MNRAS, 358, 1069

[24] Khangulyan, D., Hnatic, S., Aharonian, F., \& Bogovalov, S. 2007, MNRAS, 380, 320

[25] Khangulyan, D., Aharonian, F., \& Bosch-Ramon, V. 2008, MNRAS, 383, 467

[26] Liu, Q. Z., van Paradijs, J., \& van den Heuvel, E. P. J. 2006, A\&A, 455, 1165

[27] Liu, Q. Z., van Paradijs, J., \& van den Heuvel, E. P. J. 2007, A\&A, 469, 807

[28] Maier, G. (for the VERITAS Collaboration) 2007, Proc. of the 30th International Cosmic Ray Conference (Mérida), in press, [arXiv:0709.3661v1]

[29] Martí, J., Paredes, J. M., \& Ribó, M. 1998, A\&A, 338, L71

[30] Massi, M., Ribó, M., Paredes, J. M., et al. 2004, A\&A, 414, L1

[31] Mirabel, I. F., \& Rodríguez, L. F. 1999, ARA\&A, 37, 409

[32] Neronov, A., \& Chernyakova, M. 2007, Ap\&SS, 309, 253

[33] Neronov, A., \& Chernyakova, M. 2008, ApJ, 672, L123

[34] Paredes, J. M., Martí, J., Ribó, M., \& Massi, M. 2000, Science, 288, 2340

[35] Paredes, J. M., Ribó, M., Ros, E., Martí, J., \& Massi, M. 2002, A\&A, 393, L99

[36] Paredes, J. M., Bosch-Ramon, V., \& Romero, G. E. 2006, A\&A, 451, 259

[37] Ribó, M., Reig, P., Martí, J., \& Paredes, J. M. 1999, A\&A, 347, 518

[38] Ribó, M., Paredes, J. M., Romero, G. E., et al. 2002, A\&A, 384, 954

[39] Ribó, M., Paredes, J. M., Moldón, J. Martí, J., \& Massi, M. 2008, A\&A, in press 
[40] Romero, G. E., Okazaki, A. T., Orellana, M., \& Owocki, S. P. 2007, A\&A, 474, 15

[41] Rovero, A. C., Fegan, S., \& Weekes, T. C. 2002, BAAA, 45, 66

[42] Sidro, N., Bosch-Ramon, V., Cortina, J., et al. (for the MAGIC Collaboration) 2007, Proc. of the 30th International Cosmic Ray Conference (Mérida), in press

[43] Sierpowska-Bartosik, A., \& Torres, D. F. 2007, ApJ, 671, L145

[44] Sierpowska-Bartosik, A., \& Torres, D. F. 2008, ApJ, in press, [arXiv: $0801.1487 \mathrm{v} 1]$

[45] Stirling, A. M., Spencer, R. E., de la Force, C. J., et al. 2001, MNRAS, 327, 1273

[46] Weekes, T. C. 2006, in Proceedings of the International Workshop on "Energy Budget in the High Energy Universe", Kashiwa, Japan, February 22-24, 2006, [arXiv: astro-ph / 0606130 ]

[47] Weekes, T. C., Cawley, M. F., Fegan, D. J., et al. 1989, ApJ, 342, 379

[48] Ziółkowski, J. 2005, MNRAS, 358, 851 